\title{
The existence of solution for boundary value problems for differential equations with deviating arguments and $p$-Laplacian
}

\author{
by Bing Liu and Jianshe Yu (Changsha)
}

\begin{abstract}
We consider a boundary value problem for a differential equation with deviating arguments and $p$-Laplacian: $-\left(\phi_{p}\left(x^{\prime}\right)\right)^{\prime}+\frac{d}{d t} \operatorname{grad} F(x)+g\left(t, x(t), x(\delta(t)), x^{\prime}(t)\right.$, $\left.x^{\prime}(\tau(t))\right)=0, t \in[0,1] ; x(t)=\varphi(t), t \leq 0 ; x(t)=\bar{\varphi}(t), t \geq 1$. An existence result is obtained with the help of the Leray-Schauder degree theory, with no restriction on the damping forces $\frac{d}{d t} \operatorname{grad} F(x)$.
\end{abstract}

1. Introduction. The main purpose of the present paper is to get the solvability of the following boundary value problem (BVP for short) for a differential equation with deviating arguments and $p$-Laplacian:

$$
\begin{gathered}
-\left(\phi_{p}\left(x^{\prime}\right)\right)^{\prime}+\frac{d}{d t} \operatorname{grad} F(x) \\
+g\left(t, x(t), x(\delta(t)), x^{\prime}(t), x^{\prime}(\tau(t))\right)=0, \quad t \in[0,1], \\
x(t)=\underline{\varphi}(t), \quad t \leq 0, \\
x(t)=\bar{\varphi}(t), \quad t \geq 1,
\end{gathered}
$$

where $F: \mathbb{R}^{n} \rightarrow \mathbb{R}$ is a twice continuously differentiable function, $g:[0,1] \times$ $\left(\mathbb{R}^{n}\right)^{4} \rightarrow \mathbb{R}^{n}$ is a Carathéodory function, $\delta, \tau:[0,1] \rightarrow \mathbb{R}$ are differentiable functions such that $\{t \in[0,1]: \delta(t)=0$ or $\tau(t)=1\}$ is finite and $\phi_{p}: \mathbb{R}^{n} \rightarrow$ $\mathbb{R}^{n}$ is defined by

$$
\phi_{p}(x)=\phi_{p}\left(x_{1}, \ldots, x_{n}\right)=\left(\left|x_{1}\right|^{p-2} x_{1}, \ldots,\left|x_{n}\right|^{p-2} x_{n}\right)
$$

where $1<p<\infty$. Note that $\phi_{p}$ is a homeomorphism of $\mathbb{R}^{n}$ with inverse $\phi_{q}$ $(1 / q+1 / p=1)$. Moreover, we suppose that

2000 Mathematics Subject Classification: Primary 34K10, 34L30.

Key words and phrases: boundary value problems, differential equations with deviating arguments, Leray-Schauder degree, a priori bounds, existence theorems, $p$-Laplacian.

This project is supported by NNSF of China (No. 19831030). 


$$
-\infty<-r=\min _{t \in[0,1]}\{\delta(t), \tau(t)\}<0 \quad \text { and } \quad 1<\max _{t \in[0,1]}\{\delta(t), \tau(t)\}=d<\infty,
$$

and $\varphi:[-r, 0] \rightarrow \mathbb{R}^{n}$ and $\bar{\varphi}:[1, d] \rightarrow \mathbb{R}^{n}$ are continuously differentiable functions.

By a solution $x$ of the BVP $(1),(2)$ we mean that $x \in C^{1}\left([-r, d], \mathbb{R}^{n}\right)$ and $\phi_{p}\left(x^{\prime}\right)$ is absolutely continuous on $[0,1],\left.x\right|_{[0,1]}$ satisfies the equation (1) and $\left.x\right|_{[-r, 0]}=\underline{\varphi},\left.x\right|_{[1, d]}=\bar{\varphi}$.

When $p=2$ or $\phi_{p}(x)=x$, the above BVP was recently studied by Tsamatos and Ntouyas [5] by using the Topological Transversality Method. However, the existence results in [5] mainly depend upon a strict damping force condition, i.e., there exists a nonnegative constant $Q$ such that

$$
\langle A(u) v, v\rangle \leq Q|v|^{2} \quad \text { for all } u, v \text { in } \mathbb{R}^{n}
$$

where $A$ is the Hessian matrix of $F$, and $|\cdot|$ and $\langle\cdot, \cdot\rangle$ denote the Euclidean norm and Euclidean inner product on $\mathbb{R}^{n}$ respectively. When no damping is present in (1), i.e., $F(x) \equiv 0$ and $p=2$, the above BVP $(1),(2)$ is also considered by Tsamatos and Ntouyas [6]. It is therefore natural to ask whether one can obtain an existence result with no restriction on the damping forces $\frac{d}{d t} \operatorname{grad} F(x)$. In this paper, we establish an existence result which can be applied to any damping forces without imposing more conditions on $g$. Moreover, the general exponent $p$ is allowed, and our results seem to be new even if $p=2$.

We remark that a number of studies are concerned with boundary value problems for differential equations with deviating argument by means of the Leray-Schauder Alternative Theorem (see for example [1-4]). The key tool in our approach is the Leray-Schauder degree theory. This method reduces the problems of existence of a solution for the BVP (1), (2) to establishing suitable a priori bounds for the solutions.

Throughout this paper, we assume that

$$
\underline{\varphi}(0)=\bar{\varphi}(1)=0,
$$

but this restriction is no loss of generality, since an appropriate change of variables reduces the problem with $\underline{\varphi}(0) \bar{\varphi}(1) \neq 0$ to this case.

Furthermore, the function $g:[0,1] \times\left(\mathbb{R}^{n}\right)^{4} \rightarrow \mathbb{R}^{n}$ is a Carathéodory function, which means:

(i) for almost every $t \in[0,1]$ the function $g(t, \cdot, \cdot, \cdot, \cdot)$ is continuous;

(ii) for every $(x, y, u, v) \in\left(\mathbb{R}^{n}\right)^{4}$ the function $f(\cdot, x, y, u, v)$ is measurable on $[0,1]$;

(iii) for each $\varrho>0$ there is $\bar{g}_{\varrho} \in L^{1}([0,1], \mathbb{R})$ such that, for almost every $t \in[0,1]$ and $[x, y, u, v] \in\left(\mathbb{R}^{n}\right)^{4}$ with $|x| \leq \varrho,|y| \leq \varrho,|u| \leq \varrho,|v| \leq \varrho$, one has

$$
|g(t, x, y, u, v)| \leq \bar{g}_{\varrho}(t)
$$


2. Main results. In what follows, we denote the Euclidean inner product in $\mathbb{R}^{n}$ by $\langle\cdot, \cdot\rangle$, and the $l^{p}$-norm in $\mathbb{R}^{n}$ by $|\cdot|$, i.e.

$$
|x|=\left|\left(x_{1}, \ldots, x_{n}\right)\right|=\left(\sum_{i=1}^{n}\left|x_{i}\right|^{p}\right)^{1 / p} .
$$

The corresponding $L^{p}$-norm in $L^{p}\left([0,1], \mathbb{R}^{n}\right)$ is defined by

$$
\|x\|_{p}=\left(\sum_{i=1}^{n} \int_{0}^{1}\left|x_{i}(t)\right|^{p} d t\right)^{1 / p} .
$$

The $L^{\infty}$-norm in $L^{\infty}\left([0,1], \mathbb{R}^{n}\right)$ is

$$
\|x\|_{\infty}=\max _{1 \leq i \leq n}\left\|x_{i}\right\|_{\infty}=\max _{1 \leq i \leq n} \sup _{t \in[0,1]}\left|x_{i}(t)\right| .
$$

Now, we introduce the space

$$
X=C\left([-r, d], \mathbb{R}^{n}\right) \cap C^{1}\left([-r, 0] \cup[1, d], \mathbb{R}^{n}\right) \cap C_{0}^{1}\left([0,1], \mathbb{R}^{n}\right)
$$

with the norm

$$
\|x\|_{*}=\max \left\{\|x\|_{\infty},\|x\|_{-},\|x\|_{+},\left\|x^{\prime}\right\|_{-},\left\|x^{\prime}\right\|_{+},\left\|x^{\prime}\right\|_{\infty}\right\}
$$

where

$$
\begin{gathered}
C_{0}^{1}\left([0,1], \mathbb{R}^{n}\right)=\left\{x \in C^{1}\left([0,1], \mathbb{R}^{n}\right): x(0)=x(1)=0\right\}, \\
\|x\|_{-}=\max _{1 \leq i \leq n}\left\|x_{i}\right\|_{-}=\max _{1 \leq i \leq n} \sup _{t \in[-r, 0]}\left|x_{i}(t)\right|, \\
\|x\|_{+}=\max _{1 \leq i \leq n}\left\|x_{i}\right\|_{+}=\max _{1 \leq i \leq n} \sup _{t \in[1, d]}\left|x_{i}(t)\right| .
\end{gathered}
$$

Moreover

$$
Z=L^{1}\left([0,1], \mathbb{R}^{n}\right) .
$$

Define the $p$-Laplacian $\Delta_{p}$ : dom $\Delta_{p} \subset X \rightarrow Z$ by

$$
\left(\Delta_{p} x\right)(t)=\left(\phi_{p}\left(x^{\prime}(t)\right)\right)^{\prime}
$$

where dom $\Delta_{p}=\left\{x \in X: \phi_{p}\left(x^{\prime}\right)\right.$ is absolutely continuous on $\left.[0,1]\right\}$.

Let $N: X \rightarrow Z$ be the Nemytskil operator associated with $g$ :

$$
(N x)(t)=-\frac{d}{d t} \operatorname{grad} F(x)-g\left(t, x(t), x(\delta(t)), x^{\prime}(t), x^{\prime}(\tau(t))\right) .
$$

Since the operator $\Delta_{p}: \operatorname{dom} \Delta_{p} \rightarrow Z$ is invertible [7], we can define $A$ : $X \rightarrow X$ as follows:

$$
(A x)(t)= \begin{cases}\varphi(t), & t \in[-r, 0], \\ \left(-\Delta_{p}\right)^{-1}(N x)(t), & t \in[0,1], \\ \bar{\varphi}(t), & t \in[1, d] .\end{cases}
$$

Thus, the BVP (1), (2) is equivalent to solving the fixed point problem

$$
x=A x, \quad x \in X .
$$


Now, by using the same methods as in the proof of Lemmas 1 and 2 of [7], we can show

Lemma 1. The mapping $A: X \rightarrow X$ is completely continuous, i.e. $A$ is continuous and maps bounded sets to relatively compact sets.

Next, let $W^{1, p}\left([0,1], \mathbb{R}^{n}\right)$ be the Sobolev space.

Lemma 2 (see [7]). If $x \in W^{1, p}\left([0,1], \mathbb{R}^{n}\right)$ and $x(0)=x(1)=0$, then

$$
\|x\|_{p} \leq \pi_{p}^{-1}\left\|x^{\prime}\right\|_{p} \quad \text { and } \quad\|x\|_{\infty} \leq 2^{-1 / q}\left\|x^{\prime}\right\|_{p}
$$

where $1 / p+1 / q=1$ and

$$
\pi_{p}=2 \int_{0}^{(p-1)^{1 / p}} \frac{d s}{\left(1-s^{p} /(p-1)\right)^{1 / p}}=\frac{2 \pi(p-1)^{1 / p}}{p \sin (\pi / p)} .
$$

Theorem 1. Let $p>1$ be an integer. Assume that there exist constants $\delta_{0}, \tau_{0}$ such that

$$
\left|\delta^{\prime}(t)\right| \geq \delta_{0}>0 \quad \text { and } \quad\left|\tau^{\prime}(t)\right| \geq \tau_{0}>0 \quad \text { for all } t \in[0,1] .
$$

Furthermore, suppose that:

$\left(H_{1}\right)$ There exist nonnegative integers $m_{1}(<p), m_{3}(<p)$, nonnegative constants $m_{2}(<p), \theta(<p), \bar{a}, \bar{b}_{i}(i=1,2,3)$, and real functions $b_{i}(i=$ $1,2,3)$, c defined on $[0,1]$ with

$$
|a(t)| \leq \bar{a}, \quad\left|b_{i}(t)\right| \leq \bar{b}_{i} \quad(i=1,2,3)
$$

for all $t \in[0,1], c \in L^{1}([0,1], \mathbb{R})$ and such that

$$
\left\langle x, g\left(t, x, u_{1}, u_{2}, u_{3}\right)\right\rangle \geq a(t)|x|^{p}+\sum_{i=1}^{3} b_{i}(t)|x|^{p-m_{i}}\left|u_{i}\right|^{m_{i}}+c(t)|x|^{\theta}
$$

for all $x, u_{1}, u_{2}, u_{3} \in \mathbb{R}^{n}$ and almost $t \in[0,1]$.

$\left(H_{2}\right)$ There exist constants $\alpha \geq 0, \beta \geq 0$, a nonnegative integer $n_{1}(<p)$, $h \in L^{1}\left([0,1], \mathbb{R}_{+}\right)$, and a Carathéodory function $G:[0,1] \times\left(\mathbb{R}^{n}\right)^{2} \rightarrow \mathbb{R}^{n}$ such that

$$
|g(t, x, u, v, w)| \leq|G(t, x, u)|+\alpha|v|^{p}+\beta|v|^{p-n_{1}}|w|^{n_{1}}+h(t)
$$

for all $x, u, v, w \in \mathbb{R}^{n}$ and almost all $t \in[0,1]$.

Then the BVP (1), (2) has at least one solution provided that

$$
\bar{a}+\bar{b}_{1} \delta_{0}^{-m_{1} / p}+\bar{b}_{2} \pi_{p}^{m_{2}}+\bar{b}_{3} \tau_{0}^{-m_{3} / p} \pi_{p}^{m_{3}}<\pi_{p}^{p}
$$

where $\pi_{p}$ is defined by (4). 
Pr o of. Consider the auxiliary BVP

$(5)$

$$
\left\{\begin{array}{l}
-\left(\phi_{p}\left(x^{\prime}\right)\right)^{\prime}+\lambda \frac{d}{d t} \operatorname{grad} F(x) \\
\quad+\lambda g\left(t, x(t), x(\delta(t)), x^{\prime}(t), x^{\prime}(\tau(t))\right)=0, \quad t \in[0,1], \\
x(t)=\lambda \underline{\varphi}(t), \quad t \in[-r, 0], \\
x(t)=\lambda \bar{\varphi}(t), \quad t \in[1, d],
\end{array}\right.
$$

where $\lambda \in[0,1]$. In view of the reduction from (1), (2) to (3), the BVP (5) is equivalent to the equation

$$
x=A(x, \lambda), \quad x \in X,
$$

where

$$
A(x, \lambda)(t)= \begin{cases}\lambda \underline{\varphi}(t), & t \in[-r, 0], \\ \left(-\Delta_{p}\right)^{-1}(\lambda N x)(t), & t \in[0,1], \\ \lambda \bar{\varphi}(t), & t \in[1, d] .\end{cases}
$$

First, we verify that the set of all possible solutions of the family (5) of BVPs, $\lambda \in[0,1]$, is a priori bounded by a constant independent of $\lambda$. In fact, suppose $x \in X$ is a solution of (5) for some $\lambda \in[0,1]$. Note that $x(0)=x(1)=0$. Then we get

$$
\left\|x^{\prime}\right\|_{p}^{p}=\int_{0}^{1}\left\langle x,-\left(\phi_{p}\left(x^{\prime}\right)\right)^{\prime}\right\rangle d x
$$

and

(9) $\quad \int_{0}^{1}\left\langle x, \frac{d}{d t} \operatorname{grad} F(x)\right\rangle d t=\int_{0}^{1} \frac{d}{d t}\langle x, \operatorname{grad} F(x)\rangle d t-\int_{0}^{1} \frac{d}{d t} F(x) d t=0$.

Thus, in view of $\left(H_{1}\right)$, Hölder's inequality, and (8), (9), we have

$$
\begin{aligned}
0= & \int_{0}^{1}\left\langle x,-\left(\phi_{p}\left(x^{\prime}\right)\right)^{\prime}\right\rangle d x+\lambda \int_{0}^{1}\left\langle x(t), \frac{d}{d t} \operatorname{grad} F(x)\right\rangle d t \\
& +\lambda \int_{0}^{1}\left\langle x, g\left(t, x(t), x(\delta(t)), x^{\prime}(t), x^{\prime}(\tau(t))\right)\right\rangle d t \\
= & \left\|x^{\prime}\right\|_{p}^{p}+\lambda \int_{0}^{1}\left\langle x, g\left(t, x(t), x^{\prime}(\delta(t)), x^{\prime}(t), x^{\prime}(\tau(t))\right)\right\rangle d t \\
\geq & \left\|x^{\prime}\right\|_{p}^{p}+\lambda \int_{0}^{1} a(t)|x(t)|^{p} d t+\lambda \int_{0}^{1} b_{1}(t)|x(t)|^{p-m_{1}}|x(\delta(t))|^{m_{1}} d t \\
& +\lambda \int_{0}^{1} b_{2}(t)|x(t)|^{p-m_{2}}\left|x^{\prime}(t)\right|^{m_{2}} d t
\end{aligned}
$$




$$
\begin{aligned}
& +\lambda \int_{0}^{1} b_{3}(t)|x(t)|^{p-m_{3}}\left|x^{\prime}(\tau(t))\right|^{m_{3}} d t+\lambda \int_{0}^{1} c(t)|x(t)|^{\theta} d t \\
\geq & \left\|x^{\prime}\right\|_{p}^{p}-\int_{0}^{1}|a(t)| \cdot|x(t)|^{p} d t-\int_{0}^{1}\left|b_{1}(t)\right| \cdot|x(t)|^{p-m_{1}}|x(\delta(t))|^{m_{1}} d t \\
& -\int_{0}^{1}\left|b_{2}(t)\right| \cdot|x(t)|^{p-m_{2}}\left|x^{\prime}(t)\right|^{m_{2}} d t \\
& -\int_{0}^{1}\left|b_{3}(t)\right| \cdot|x(t)|^{p-m_{3}}\left|x^{\prime}(\tau(t))\right|^{m_{3}} d t-\|x\|_{\infty}^{\theta} \int_{0}^{1}|c(t)| d t \\
\geq & \left\|x^{\prime}\right\|_{p}^{p}-\bar{a} \int_{0}^{1}|x(t)|^{p} d t-\bar{b}_{1}^{1}|x(t)|^{p-m_{1}}|x(\delta(t))|^{m_{1}} d t \\
& -\bar{b}_{2} \int_{0}^{1}|x(t)|^{p-m_{2}}\left|x^{\prime}(t)\right|^{m_{2}} d t \\
& -\bar{b}_{3} \int_{0}^{1}|x(t)|^{p-m_{3}}\left|x^{\prime}(\tau(t))\right|^{m_{3}} d t-\|x\|_{\infty}^{\theta}\|c\|_{1} \\
\geq & \left\|x^{\prime}\right\|_{p}^{p}-\bar{a}\|x\|_{p}^{p}-\bar{b}_{1}\|x\|_{p}^{p-m_{1}}\left(\int_{0}^{1}|x(\delta(t))|^{p} d t\right)^{m_{1} / p} \\
& -\bar{b}_{2}\|x\|_{p}^{p-m_{2}}\left\|x^{\prime}\right\|_{p}^{m_{2}}{ }_{0}^{1} \mid \bar{b}_{3}\|x\|_{p}^{p-m_{3}}\left(\left.\int_{0}^{\prime}(\tau(t))\right|^{p} d t\right)^{m_{3} / p}-\|x\|_{\infty}^{\theta}\|c\|_{1} \cdot \\
&
\end{aligned}
$$

Again

$$
\begin{aligned}
& \left(\int_{0}^{1}|x(\delta(t))|^{p} d t\right)^{m_{1} / p} \\
& =\left[\int_{0}^{1}|x(\delta(t))|^{p} \cdot \frac{1}{\delta^{\prime}(t)} d(\delta(t))\right]^{m_{1} / p} \leq \delta_{0}^{-m_{1} / p}\left[\int_{\delta([0,1])}|x(s)|^{p} d s\right]^{m_{1} / p} \\
& =\delta_{0}^{-m_{1} / p}\left[\int_{0}^{1}|x(s)|^{p} d t+\int_{-r}^{0}|x(s)|^{p} d s+\int_{1}^{d}|x(s)|^{p} d s\right]^{m_{1} / p} \\
& =\delta_{0}^{-m_{1} / p}\left[\|x\|_{p}^{p}+\left.\int_{-r}^{\mid \varphi}(t)\right|^{p} d s+\int_{1}^{d}|\bar{\varphi}(s)|^{p} d s\right]^{m_{1} / p} \\
& =\delta_{0}^{-m_{1} / p}\left[\|x\|_{p}^{p}+\Delta_{1}^{p}\right]^{m_{1} / p} \leq \delta_{0}^{-m_{1} / p}\left[\|x\|_{p}+\Delta_{1}\right]^{m_{1}} \\
& =\delta_{0}^{-m_{1} / p}\left[\|x\|_{p}^{m_{1}}+\sum_{k=1}^{m_{1}}\left(\begin{array}{c}
m_{1} \\
k
\end{array}\right)\|x\|_{p}^{m_{1}-k} \Delta_{1}^{k}\right]
\end{aligned}
$$


where $\Delta_{1}=\left(\int_{-r}^{0}|\underline{\varphi}(s)|^{p} d s+\int_{1}^{d}|\bar{\varphi}(s)|^{p} d s\right)^{1 / p}$. Similarly

$$
\left(\int_{0}^{1}\left|x^{\prime}(\tau(t))\right|^{p} d t\right)^{m_{3} / p} \leq \tau_{0}^{-m_{3} / p}\left[\left\|x^{\prime}\right\|_{p}^{m_{3}}+\sum_{k=1}^{m_{3}}\left(\begin{array}{c}
m_{3} \\
k
\end{array}\right)\left\|x^{\prime}\right\|_{p}^{m_{3}-k} \Delta_{2}^{k}\right]
$$

where $\Delta_{2}=\left(\int_{-r}^{0}\left|\underline{\varphi^{\prime}}(s)\right|^{p} d s+\int_{1}^{d}\left|\bar{\varphi}^{\prime}(s)\right|^{p} d s\right)^{1 / p}$. From (10)-(12) and Lemma 2 , we obtain

$$
\begin{aligned}
0 \geq & \left\|x^{\prime}\right\|_{p}^{p}-\bar{a}\|x\|_{p}^{p}-\bar{b}_{1} \delta_{0}^{-m_{1} / p}\left[\|x\|_{p}^{p}+\sum_{k=1}^{m_{1}}\left(\begin{array}{c}
m_{1} \\
k
\end{array}\right)\|x\|_{p}^{p-k} \Delta_{1}^{k}\right] \\
& -\bar{b}_{2}\|x\|_{p}^{p-m_{2}}\left\|x^{\prime}\right\|_{p}^{m_{2}} \\
& -\bar{b}_{3} \tau_{0}^{-m_{3} / p}\|x\|_{p}^{p-m_{3}}\left[\left\|x^{\prime}\right\|_{p}^{m_{3}}+\sum_{k=1}^{k}\left\|x^{\prime}\right\|_{p}^{m_{3}-k} \Delta_{2}^{k}\right]-\|x\|_{\infty}^{\theta}\|c\|_{1} \\
\geq & \left\|x^{\prime}\right\|_{p}^{p}-\bar{a} \pi_{p}^{-p}\left\|x^{\prime}\right\|_{p}^{p}-\bar{b}_{1} \delta_{0}^{-m_{1} / p} \pi_{p}^{-p}\left\|x^{\prime}\right\|_{p}^{p}-\bar{b}_{2} \pi_{p}^{m_{2}-p}\left\|x^{\prime}\right\|_{p}^{p} \\
& -\bar{b}_{3} \tau_{0}^{-m_{3} / p} \pi_{p}^{m_{3}-p}\left\|x^{\prime}\right\|_{p}^{p}-\bar{b}_{1} \delta_{0}^{-m_{1} / p} \sum_{k=1}^{m_{1}}\left(\begin{array}{c}
m_{1} \\
k
\end{array}\right) \Delta_{1}^{k} \pi_{p}^{k-p}\left\|x^{\prime}\right\|_{p}^{p-k} \\
& -\bar{b}_{3} \tau_{0}^{-m_{3} / p} \pi_{p}^{m_{3}-p} \sum_{k=1}^{m_{3}}\left(\begin{array}{c}
m_{3} \\
k
\end{array}\right) \Delta_{2}^{k}\left\|x^{\prime}\right\|_{p}^{p-k}-2^{-1 / q}\|c\|_{1}\left\|x^{\prime}\right\|_{p}^{\theta},
\end{aligned}
$$

which yields

$$
\begin{aligned}
\left\|x^{\prime}\right\|_{p}^{p} \leq & \frac{1}{\Lambda}\left[\bar{b}_{1} \delta_{1}^{-m_{1} / p} \sum_{k=1}^{m_{1}}\left(\begin{array}{c}
m_{1} \\
k
\end{array}\right) \Delta_{1}^{k} \pi_{p}^{k-p}\left\|x^{\prime}\right\|_{p}^{p-k}\right. \\
& +\bar{b}_{3} \tau_{0}^{-m_{3} / p} \pi_{p}^{m_{3}-p} \sum_{k=1}^{m_{3}}\left(\begin{array}{c}
m_{3} \\
k
\end{array}\right) \Delta_{2}^{k}\left\|x^{\prime}\right\|_{p}^{p-k} \\
& \left.+2^{-1 / q}\|c\|_{1}\left\|x^{\prime}\right\|_{p}^{\theta}\right]
\end{aligned}
$$

where

$$
\Lambda=1-\left[\bar{a}+\bar{b}_{1} \delta_{0}^{-m_{1} / p}+\bar{b}_{2} \pi_{p}^{m_{2}}+\bar{b}_{3} \tau_{0}^{-m_{3} / p} \pi_{p}^{m_{3}}\right] \pi_{p}^{-p}>0 .
$$

Since $m_{1}<p, m_{3}<p, \theta<p$, from (13) we see that there exists a constant $M>0$ such that

$$
\left\|x^{\prime}\right\|_{p} \leq M
$$

Hence by Lemma 2, there exists a constant $M_{1}=2^{-1 / q} M$ such that

$$
\|x\|_{\infty} \leq M_{1}
$$

By $(15),|x(t)|=\left(\sum_{i=1}^{n}\left|x_{i}(t)\right|^{p}\right)^{1 / p}$ is bounded, thus since $F \in C^{2}\left(\mathbb{R}^{n}, \mathbb{R}\right)$, there exists a constant $M_{2}>0$ such that $\left|\frac{\partial^{2} F(x)}{\partial x^{2}}\right| \leq M_{2}$. Therefore, from 
$\left(H_{2}\right)$ and (15), we have

$$
\begin{aligned}
\int_{0}^{1}\left|\left(\phi_{p}\left(x^{\prime}\right)\right)^{\prime}\right| d t= & \lambda \int_{0}^{1}\left|\frac{d}{d t} \operatorname{grad} F(x)+g\left(t, x(\delta(t)), x^{\prime}(t), x^{\prime}(\tau(t))\right)\right| d t \\
\leq & \int_{0}^{1}\left|\frac{\partial^{2} F(x)}{\partial x^{2}}\right|\left|x^{\prime}\right| d t+\int_{0}^{1}|G(t, x(t)), x(\delta(t))| d t \\
& +\alpha \int_{0}^{1}\left|x^{\prime}(t)\right|^{p} d t \\
& +\beta \int_{0}^{1}\left|x^{\prime}(t)\right|^{p-n_{1}}\left|x^{\prime}(\tau(t))\right|^{n_{1}} d t+\int_{0}^{1} h(t) d t \\
\leq & \int_{0}^{1} \bar{G}_{\varrho}(t) d t+M_{2}\left\|x^{\prime}\right\|_{p}+\alpha\left\|x^{\prime}\right\|_{p}^{p} \\
& +\beta\left\|x^{\prime}\right\|_{p}^{p-n_{1}}\left(\int_{0}^{1}\left|x^{\prime}(\tau(t))\right|^{p} d t\right)^{n_{1} / p}+\|h\|_{1}
\end{aligned}
$$

where $\varrho=\max \left\{M_{1},\|\underline{\varphi}\|_{-},\|\varphi\|_{+}\right\}$, and $\bar{G}_{\varrho} \in L^{1}([0,1], \mathbb{R})$ is such that

$$
|G(t, x, y)| \leq \bar{G}_{\varrho}(t)
$$

when $|x| \leq \varrho,|y| \leq \varrho$. The existence of $\bar{G}_{\varrho}$ is guaranteed by the fact that $G$ is of Carathéodory type.

Similarly to (13), we have

$$
\left(\int_{0}^{1}\left|x^{\prime}(\tau(t))\right|^{p} d t\right)^{n_{1} / p} \leq \tau_{0}^{-n_{1} / p}\left[\left\|x^{\prime}\right\|_{p}^{n_{1}}+\sum_{k=1}^{n_{1}}\left(\begin{array}{c}
n_{1} \\
k
\end{array}\right)\left\|x^{\prime}\right\|_{p}^{n_{1}-k} \Delta_{2}^{k}\right] .
$$

Thus from (14), (16), (17) one has

$$
\begin{aligned}
\int_{0}^{1}\left|\left(\phi_{p}\left(x^{\prime}\right)\right)^{\prime}\right| d t \leq & \int_{0}^{1} \bar{G}_{\varrho}(t) d t+M_{2} M+\alpha M^{p} \\
& +\beta \tau_{0}^{-n_{1} / p}\left[M^{p}+\sum_{k=1}^{n_{1}}\left(\begin{array}{c}
n_{1} \\
k
\end{array}\right) \Delta_{2}^{k} M^{n_{1}-k}\right]=M_{3} .
\end{aligned}
$$

Again for each $i=1, \ldots, n$, as $x_{i}(0)=x_{i}(1)=0$, we have $x_{i}^{\prime}\left(t_{i}\right)=0$ for some $t_{i} \in(0,1)$. Thus for any $t \in[0,1]$, we obtain

$$
\left|\phi_{p}\left(x_{i}^{\prime}(t)\right)\right|=\left|\phi_{p}\left(x_{i}^{\prime}(t)\right)-\phi_{p}\left(x_{i}^{\prime}\left(t_{i}\right)\right)\right|=\left|\int_{t_{i}}^{t}\left(\phi_{p}\left(x_{i}^{\prime}(s)\right)\right)^{\prime} d s\right| \leq M_{3} .
$$

Hence for all $i \in\{1, \ldots, n\}$ and $t \in[0,1]$, one has $\left|x_{i}^{\prime}(t)\right| \leq \phi_{q}\left(M_{3}\right)$, which 
yields $\left\|x^{\prime}\right\|_{\infty} \leq \phi_{q}\left(M_{3}\right)$. Thus, from (15) we have

$$
\begin{aligned}
\|x\|_{*} & =\max \left\{\|x\|_{\infty},\|x\|_{-},\|x\|_{+},\left\|x^{\prime}\right\|_{-},\left\|x^{\prime}\right\|_{+},\left\|x^{\prime}\right\|_{\infty}\right\} \\
& \leq \max \left\{M_{1},\|\underline{\varphi}\|_{-},\|\bar{\varphi}\|_{+},\left\|\underline{\varphi^{\prime}}\right\|_{-},\left\|\bar{\varphi}^{\prime}\right\|_{+}, \phi_{q}\left(M_{3}\right)\right\}=M_{4},
\end{aligned}
$$

which implies $\|x\|_{*}$ is bounded.

Next, taking $r>M_{4}$, set

$$
\Omega=\left\{x \in X:\|x\|_{*}<r\right\} .
$$

From the above argument, (6) does not have a solution for $(x, \lambda) \in \partial \Omega \times$ $[0,1]$. Thus for each $\lambda \in[0,1]$, the Leray-Schauder degree $\operatorname{deg}_{\mathrm{LS}}[I-A(\cdot, \lambda)$, $\Omega, 0]$ is well defined and by the properties of that degree,

$$
\operatorname{deg}_{\mathrm{LS}}[I-A(\cdot, 1), \Omega, 0]=\operatorname{deg}_{\mathrm{LS}}[I-A(\cdot, 0), \Omega, 0] .
$$

Now it is clear that the problem

$$
x=A(x, 1)
$$

is equivalent to (3). Since $A(x, 0)=0$ for all $x \in \Omega$, from (18) we have

$$
\operatorname{deg}_{\mathrm{LS}}[I-A(\cdot, 1), \Omega, 0]=\operatorname{deg}_{L S}[I, \Omega, 0] \neq 0,
$$

which yields that the problem (19), and hence (3), has a solution, so that the BVP (1), (2) has at least one solution. This completes the proof.

REMARK 1. Similar results can be obtained for the BVP

$$
\left\{\begin{array}{l}
-\left(\phi_{p}\left(x^{\prime}\right)\right)^{\prime}+\frac{d}{d t} \operatorname{grad} F(x)+g\left(t, x(t), x\left(\delta_{1}(t)\right), \ldots, x\left(\delta_{k}(t)\right),\right. \\
\left.\quad x^{\prime}(t), x^{\prime}\left(\tau_{1}(t)\right), \ldots, x^{\prime}\left(\tau_{l}(t)\right)\right)=0, \quad t \in[0,1], \\
x(t)=\varphi(t), \quad t \leq 0, \\
x(t)=\overline{\bar{\varphi}}(t), \quad t \geq 1 .
\end{array}\right.
$$

REMARK 2. The BVP

$$
\begin{cases}\left(\phi_{p}\left(x^{\prime}\right)\right)^{\prime}+\frac{d}{d t} \operatorname{grad} F(x) & \\ \quad+g\left(t, x(t), x(\delta(t)), x^{\prime}(t), x^{\prime}(\tau(t))\right)=0, \quad t \in[0,1], \\ x(t)=\varphi(t), & t \leq 0, \\ x(t)=\overline{\bar{\varphi}}(t), & t \geq 1,\end{cases}
$$

can also be studied by the methods of this paper.

\section{References}

[1] J. Lee and D. O'Regan, Existence results for differential delay equations I, J. Differential Equations 102 (1993), 342-359.

[2] - - - Existence results for differential delay equations II, Nonlinear Anal. 17 (1991), 683-902. 
[3] B. Liu and J. S. Yu, Note on a third order boundary value problem for differential equations with deviating arguments, preprint.

[4] S. N touy as and P. Tsamatos, Existence and uniqueness for second order boundary value problems, Funkcial. Ekvac. 38 (1995), 59-69.

[5] - - - Existence and uniquenes of solutions for boundary value problems for differential equations with deviating arguments, Nonlinear Anal. 22 (1994), 113-1-1146.

[6] - - - Existence of solutions of boundary value problems for differential equations with deviating arguments, via the topological transversality method, Proc. Roy. Soc. Edinburgh Sect. A 118 (1991), 79-89.

[7] M. R. Zhang, Nonuniform nonresonance at the first eigenvalue of the p-Laplacian, Nonlinear Anal. 29 (1997), 41-51.

Department of Applied Mathematics

Hunan University

Changsha 410082

People's Republic of China

E-mail: jsyu@mail.hunu.edu.cn

Reçu par la Rédaction le 27.4.2000 